\title{
Transport determinants for continuing care residents assessed by an EMS urgent response team: A retrospective observational study
}

\author{
By Kevin Lobay, DMD, MD, MBA, Robyn Palmer, MD, Lorissa Mews, MD, Robert Sharman RN, EMT-P, BHScN, \\ MA, Brian Boswell, EMT-P, and Priya Jaggi, MSc
}

\begin{abstract}
Introduction: Alberta Health Services (AHS) Emergency Medical Services (EMS) in the City of Edmonton recently introduced an "EMS Continuing Care Urgent Response Team" (ECCURT) to support continuing care residents by providing urgent care on-site, thereby minimizing unnecessary patient transfers to emergency departments. ECCURT is comprised of Advanced Care Paramedics and Nurse Practitioners, and is dispatched via a dedicated consult line and/or 911.
\end{abstract}

Objectives: This study will identify various patient characteristics that are correlated with frequency of transport to hospital.

Methods: This six-month retrospective, observational study of patient data includes all new patients assessed between January 1, 2016 and June 30, 2016. Multiple regression analysis was performed to determine whether a statistically significant correlation exists comparing age, Goals of Care Designation (GCD), and Canadian Triage Acuity Scale (CTAS) score, with transport frequency.

Results: Four hundred and seventy-one (83\%) of 567 new patients assessed by ECCURT during the study period had established GCDs in place. Five hundred and twenty-one (92\%) of our patients had a CTAS score assigned. One hundred and thirty-one (23\%) of our patients were transported to hospital. All patients with a GCD of C2 were managed by our team on-site. Multiple regression analysis reveals a statistically significant correlation of age, GCD, and CTAS score with frequency of transport to hospital (F statistic $=3.26 \mathrm{E}-11)$. P-values for each variable are: age $=0.92 ; \mathrm{GCD}=0.05 ; \mathrm{CTAS}=5.08 \mathrm{E}-12$.

Conclusion: Although patient age is not strongly correlated with transport frequency independently, GCD and
CTAS score may be quite useful predictors for Community Care EMS Teams when selecting patients who can be managed on-site without transport to hospital.

Key words: urgent response team; paramedics; nurse practitioners; Goals of Care Designation; CTAS Score

\section{Introduction}

$\mathrm{M}$ any of the interventions provided during an Emergency Department (ED) visit can be performed within a continuing care facility, including therapies such as IV catheter placement with fluid administration, urethral catheter placement, oxygen administration, and oral or intravenous medication administration (Ackermann, 2001; Ackermann, 1998; Trahan, Spiers, \& Cummings, 2016). When transported to hospital, patients with these needs often return to their residence following ED assessment and treatment (Reid, et al., 2013). Treatment performed safely within a patient's home, rather than in hospital, may comply with patient wishes and protect them from inherent risks of hospitalization such as hospital-acquired infections and risk of delirium (Caplan, Meller, Squires, Chan, \& Willett, 2006; Fong, et al., 2012; Nyweide, Anthony, Bynum, et al., 2013).

The continuing care population is characterized by a high proportion of frail elderly patients with complex medical needs (Canadian Institute for Health Information, 2016). To serve this population in Edmonton, an EMS Continuing Care Urgent Response Team (ECCURT) was introduced by Alberta Health Services (AHS) Emergency Medical Services (EMS). ECCURT personnel include Nurse Practitioners and Advanced Care Paramedics who can assess and initiate treatment for patients in consultation with family physicians. ECCURT vehicles are equipped with standard ambulance resources and additional tools including blood gas analyzers, urinary catheters, splinting materials, sutures, intravenous antibiotics and various oral medications. On-site use of these tools can mitigate transport to hospital of many patients with lower acuity medical needs.

\section{Objectives}

This study provides an overview of the patients who are assessed and managed by ECCURT, and determines whether a 
statistically significant correlation exists comparing patient age, Goals of Care Designation (GCD), and CTAS score with frequency of transport to hospital.

\section{Methods}

Research setting: Our study was conducted in the city of Edmonton, which is served by a unified 911 dispatch and ambulance service (AHS EMS). ECCURT responds to all 63 supportive-living and long-term care facilities in Edmonton, housing approximately 6894 clients.

Design: This retrospective, observational study provides an overview of ECCURT patient demographics, and relates age, GCD and CTAS score with frequency of transport to hospital using multiple regression analysis. Our study was approved by the University of Alberta Health Research Ethics Board (Panel B), and by the AHS EMS Research Committee. The authors have no conflicts of interest to declare.

Study population: All patients living in Edmonton Continuing Care facilities who were assessed by ECCURT between 1-January-2016 and 30-June-2016 are included. For patients assessed multiple times, repeat visits within a 1-month period, and repeat visits for ongoing management, were excluded from analysis.

Data collection: For all patients, the following variables were recorded in our internal Microsoft Access Database: age, CTAS score assigned on initial patient encounter, presence/level of GCD, ICD-9 diagnosis assigned upon completion of the patient encounter, and whether the patient required transport to an ED for further investigations and/or treatment.

Analysis: Statistical analysis was performed by the authors using Microsoft Excel. We performed a multiple regression analysis comparing age, CTAS Score, and GCD with frequency of transfer to hospital.

\begin{tabular}{|c|c|c|c|}
\hline GCD & $\begin{array}{c}\text { Number of } \\
\text { Patients }\end{array}$ & $\begin{array}{c}\text { Number } \\
\text { Transported }\end{array}$ & \% Transported \\
\hline $\mathrm{R} 1$ & 91 & 20 & 21.98 \\
\hline R2 & 11 & 2 & 18.18 \\
\hline R3 & 23 & 5 & 21.74 \\
\hline M1 & 232 & 62 & 26.72 \\
\hline M2 & 54 & 6 & 11.10 \\
\hline $\mathrm{C} 1$ & 47 & 8 & 17.00 \\
\hline $\mathrm{C} 2$ & 13 & 0 & 0.00 \\
\hline Sum & 471 & 103 & 21.87 \\
\hline \multicolumn{4}{|c|}{$\begin{array}{l}\text { Transport Frequency (unweighted) Mean 16.67; Median } \\
\text { 18.18; Standard Deviation 8.16; } \\
\text { Simple Linear Regression Correlation: }(r) \text {-0.69; Slope -2.82; } \\
\text { Y-intercept 27.95; Sig F }(p) 0.0850\end{array}$} \\
\hline
\end{tabular}

\section{Results}

Five hundred and sixty-seven new patient visits by ECCURT occurred during the study period, and all are included in our analysis. Most of these patients were treated on site, with only a minority $(23 \%)$ requiring transport to hospital. Eighty-nine percent of our patients had a documented diagnosis. The most common diagnoses were pneumonia, musculoskeletal injury, laceration, cellulitis, fluid/electolyte abnormality (primarily dehydration), genitourinary problem NOS (primarily urinary retention), and failure to thrive.

Multiple regression analysis reveals that a statistically significant relationship exists when comparing age, GCD and CTAS score with transport frequency. P-values for each variable are: age = 0.92; GCD $=0.05$; CTAS $=5.08 \mathrm{E}-12$. $\left(\mathrm{R}^{2}\right.$ is 0.09$)$. Patient age ranged from 25 to 104 years, with a median of 84 years and a mean of 80.5 years. A poor correlation exists between age and transport frequency in our model, suggesting that age is not a good independent predictor of whether a patient will require transport to hospital. Four hundred and seventy-one $(83 \%)$ of our patients had existing GCDs. Patients with less-aggressive GCDs trend toward lower transport frequencies $(\mathrm{P}=0.05)$ (See Table and Figure). No patient with a GCD of C2 (indicating comfort-level care without transportation) was transported to hospital. Five hundred and twenty-one (92\%) of our patients were assigned CTAS scores. Patients were significantly less likely to be transferred to hospital if they had been assigned a less-acute CTAS score $(\mathrm{P}=5.08 \mathrm{E}-12)$.

\section{Discussion}

Alberta Health Services strives "to provide every patient with the right care, at the right time, by the right provider" (Alberta Health Services, 2017). ECCURT strives to meet this objective by dispatching nurse practitioners and paramedics with an enhanced scope of practice to the patient's home facility, enabling limited on-site investigations and treatment. Further analysis is needed to determine whether ECCURT programs reduce system costs, reduce ED overcrowding, and reduce morbidity and mortality associated with hospitalization.

Goals of Care documentation has become commonplace in patients whose health is in decline. Although such documentation does not consistently modify interventions performed by healthcare practitioners, a standardized form was introduced by AHS to help clarify and document interventions patients deem acceptable (Dobalian, 2004; Government of Alberta, 2013; Murray, Leblanc, $\&$ Beveridge, The Canadian Triage and Acuity Scale for Ontario paramedics, 2001; Purdy, 2002). Alberta's GCD document

\section{Figure 1.}

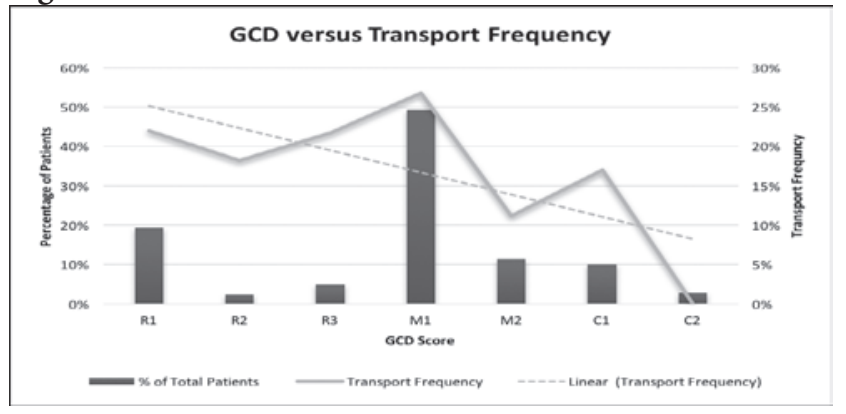


assigns an alphanumeric code to each level of therapy, which is documented in medical charts and provided to the patient.

Most patients ( $83 \%$ of our sample) living in continuing care facilities within Edmonton who are assessed by ECCURT have established GCDs. Our study suggests a trend toward decreasing transport frequencies with less-aggressive GCDs, and we believe that these scores should be considered when deciding whether a patient should be transported to hospital.

The Canadian Triage Acuity Scale (CTAS) provides a means of standardizing patients' acuity in an emergency medical environment. CTAS was introduced in Canada in 1999, and has been adopted in emergency departments worldwide, with evidence to support its reliability and validity. In 2001, CTAS was introduced for use by paramedics in Ontario (Murray, Leblanc, \& Beveridge, The Canadian Triage and Acuity Scale for Ontario paramedics, 2001). That same year, a study performed in Edmonton compared paramedic and ED triage nurse score assignment on ED arrival with moderate to good weighted Kappa agreement 0.61 (95\% CI 0.56 - 0.66) (Murray \& Bondy, 2002). Our data reveal a very significant inverse correlation between CTAS score and transport frequency. Patients with more acute CTAS scores are much less likely to be manageable on-site.

\section{Conclusion}

Our study provides useful demographic data and suggests that CTAS scores and GCDs are important factors that impact the frequency of transport to hospital. ECCURT team members and EMS medical directors wishing to build similar programs in their communities may consider incorporating these variables into their dispatch and transport decision algorithm.

\section{About the authors}

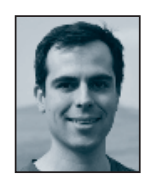

Dr. Kevin Lobay is an Emergency Medicine physician at the University of Alberta Hospital, and serves as Associate Zone EMS Medical Director for Alberta Health Services in Edmonton. Dr. Lobay also enjoys teaching part-time at the University of Alberta School of Dentistry, volunteers internationally as a general dentist, and participates in a variety of sports.

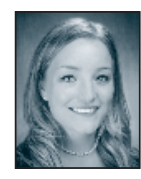

Lorissa Mews is a fifth-year FRCPC Emergency Medicine resident with a special interest in Simulation and Quality Improvement. She grew up in Ontario, attended Western University and moved to Edmonton for medical school and residency.

\section{REFERENCES}

Ackermann, R. (2001). Nursing home practice. Strategies to manage most acute and chronic illnesses without hospitalization. Geriatrics, 56(5), 37.

Ackermann, R.K. (1998). Emergency department use by nursing home residents. Annals of Emergency Medicine, 31(6), 749-757.

Alberta Health Services. (2014). Alberta Health Services. Retrieved from Goals of Care Designation http://www.albertahealthservices.ca/ frm-103547.pdf

Alberta Health Services. (2017). AHS Vision, Mission, Values and Strategies. (Alberta Health Services) Retrieved from Alberta Health Services: http://www.albertahealthservices.ca/about/ Page 190.aspx

Canadian Institute for Health Information. (2016). A snapshot of advance directives in long-term care: How often is "do not" done? Ottawa: Government of Canada.

Caplan, G., Meller, A., Squires, B., Chan, S., \& Willett, W. (2006). Advance care planning and hospital in the nursing home. Age \& Ageing, 35(6), 581-585.

Dobalian, A. (2004). Nursing facility compliance with do-nothospitalize orders. Gerontologist, 44(2), 159-165.

Fong, T., Jones, R., Marcantonio, E., Tommet, D., Gros, A., \& Habtemariam, D. (2012). Adverse outcomes after hospitalization and delirium in persons with Alzheimer disease. Annals of Internal Medicine, 156(12), 848-856.

Government of Alberta. (2013, 05 27). CanLIII. (Alberta Queen's Printer). Retrieved from Personal Directives Act https:// www.canlii.org/en/ab/laws/stat/rsa-2000-c-p-6/latest/ rsa-2000-c-p-6.html?au to complete $S$ tr $=$ personal $\% 20$ directives\&autocompletePos $=1$

Lobay, K., \& McKeown, C. (2015). Continuing Care Urgent Response Team. Edmonton: Alberta Health Services.

Murray, M., \& Bondy, S. (2002). The reliability of the Canadian Triage and Acuity Scale in the prehospital setting: Interrater reliability between paramedics and nurses. Canadian Journal of Emergency Medicine, 4, 128.

Murray, M., Leblanc, L., \& Beveridge, R.E. (2001). The Canadian Triage and Acuity Scale for Ontario paramedics. Ontario Ministry of Health and Long-Term Care, EHS Branch. Government of Ontario.

Nyweide, D., Anthony, D., Bynum, J., et al. (2013). Continuity of care and the risk of preventable hospitalization in older adults. JAMA Internal Medicine, 173(20), 1879-1885.

Purdy, W. (2002). Nursing home to emergency room? The troubling last transfer. Hastings Center Report, 32(6), 46-48.

Reid, R., Cummings, G., Cooper, S., Abel, S., Bissell, L., \& Estabrooks, C. (2013). The older persons' transitions in care (OPTIC) study: Pilot testing of the transition tracking tool. Health Services Research, 13, 515.

Trahan, L., Spiers, J., \& Cummings, G. (2016). Decisions to transfer nursing home residents to emergency department: A scoping review of contributing factors and staff perspectives. Journal of the American Medical Directors Association. Retrieved from http:// dx.doi.org/10.1016/j.jamda.2016.05.012 